\title{
Territoire et dimension géopolitique de l'accueil des réfugiés
}

\section{Les colonies agricoles des exilés du Soudan en Ouganda}

\author{
Luc Cambrézy \\ Géographe, directeur de recherche, IRD, 32 avenue Henri Varagnat, 93143 Bondy cedex, France
}

\begin{abstract}
La fréquence des interventions internationales dans le cadre de conflits locaux questionne de manière croissante le lien entre l'accueil des réfugiés et ses conséquences sur le contrôle du territoire. L'auteur montre comment une solution originale a pu être mise en œuvre en Ouganda pour l'accueil de populations en provenance du Soudan : installation dans des zones faiblement peuplées et attribution de droits - très précaires - d'usage du foncier pour la production agricole. La complexité du problème est interprétée, de même que la portée de ses dimensions économique, identitaire et environnementale. Au-delà, l'évaluation permet de questionner la capacité de l'expérience à servir de modèle, tout en posant la question fondamentale de la géopolitique de l'asile que sous-tendent de manière implicite les différentes alternatives à l'accueil des réfugiés.
\end{abstract}

La Rédaction

\section{Mots-clés :}

Réfugiés;

colonies agricoles; développement; asile ; territoire ; Ouganda

\section{Keywords:}

Keywords: Refugees; rural settlements; development; asylum; land access; Uganda

\begin{abstract}
Résumé - Contrairement au schéma classique qui consiste à endiguer les mouvements de réfugiés en regroupant ces derniers dans des camps, le gouvernement ougandais a fait le choix d'ouvrir des colonies agricoles pour les victimes de la guerre civile au Soudan. Après une analyse de cette expérience dans les deux principales zones d'accueil (Rhino Camp et district d'Adjumani), il apparaît que l'objectif recherché par le gouvernement est avant tout une opération d'aménagement et de développement du territoire avec l'appui et les moyens de l'aide humanitaire internationale. De ce point de vue, la réussite semble totale, mais survivra-t-elle au rapatriement programmé des réfugiés? On peut d'autant plus se poser la question que ce programme a été mis en œuvre dans une région peu peuplée et isolée de l'Ouganda. Dans ce contexte, la question du développement - dont les réfugiés sont ici le fer de lance - est indissociable de celle des dynamiques de peuplement et des densités de population. Mais, au-delà, le choix des lieux et des modalités de l'assistance humanitaire montre que l'aide aux exilés, dans sa dimension territoriale, est indissociable d'une réflexion sur la géopolitique de l'accueil des réfugiés.
\end{abstract}

\begin{abstract}
Spatial and geopolitical dimensions of refugee hosting. The agricultural colonies of exiled Sudanese populations in Uganda. Contrary to the commonly applied solution of controlling refugee movements by concentrating refugees in camps, the Ugandan government opted for the establishment of "rural settlements" for the population fleeing civil war in Sudan. Following a detailed analysis of this experience in two refugee hosting areas, Rhino Camp and Adjumani district, it appears clearly that the government's primary objective was a vast land planning and development operation using the means provided by international humanitarian aid. Viewed from this angle the operation seems to be a success. The question is: will this project survive the programmed repatriation of refugees? This is all the more relevant since the operation involves a low populated and isolated area of Uganda. In this context, the development issue - for which the refugees are on the frontline - cannot be disconnected from the issues of carrying capacities and population density evolution. Moreover, the sites targeted for, and modalities of humanitarian aid show that assistance to refugee populations - in its spatial aspects cannot be dissociated from another issue: the "geo-policy" of asylum.
\end{abstract}

Auteur correspondant : cambrezy@bondy.ird.fr 


\section{De l'assistance aux réfugiés à l'environnement : une recherche appliquée?}

La signature, à Nairobi en 2005, d'un accord de paix entre le gouvernement du Soudan et le mouvement de sécession du Sud du pays, dirigé, jusqu'à son récent décès, par John Garang, devrait mettre un terme à plus de 20 ans de conflit (Balancie et de la Grange, 1999). Durant cette longue période, tous les pays riverains du Soudan se sont retrouvés dans la situation de devoir accueillir $\mathrm{d}$ 'importants flux de réfugiés fuyant autant les combats entre le Nord et le Sud que les exactions de toutes sortes entre différentes communautés ethniques du Sud du pays (Lavergne, 1989). L'Éthiopie, le Kenya, l'Ouganda et, dans une moindre mesure, l'ex-Zaïre et le Tchad, ont eu à gérer, avec l'aide de la communauté internationale, l'assistance de plusieurs centaines de milliers de réfugiés en provenance du Sud-Soudan ${ }^{1}$.

L'Ouganda, comme la plupart des pays dans le monde, est lié par la convention de Genève. Cet accord vaut engagement, pour les pays signataires, d'assurer la protection et l'assistance aux populations étrangères fuyant la guerre ou les persécutions et cherchant refuge à l'étranger. Est en effet considérée comme réfugiée toute personne «craignant avec raison d'être persécutée du fait de sa race, de sa religion, de sa nationalité, de son appartenance à un certain groupe social ou de ses opinions politiques, [qui] se trouve hors du pays dont elle a la nationalité et qui ne peut ou, du fait de cette crainte, ne veut se réclamer de la protection de ce pays [...]».

Dans les pays les plus pauvres, en Afrique en particulier, il est rare que les États puissent assumer seuls la lourde charge que représente l'accueil de dizaines, voire de centaines, de milliers de réfugiés. Dans ces circonstances, les pays d'accueil font alors appel aux Nations unies par le canal du Haut Commissariat aux réfugiés (HCR), non plus seulement pour garantir et encadrer la protection des réfugiés, mais aussi pour mobiliser les moyens financiers, humains et logistiques nécessaires à l'assistance au quotidien de ces populations. C'est en général au plus haut niveau de l'État que se négocient, avec le HCR, les modalités concrètes de l'accueil. Dans la grande majorité des cas, les réfugiés sont rassemblés et accueillis dans des camps et le HCR coordonne, sous une forme contractuelle avec des ONG locales ou internationales, les diverses activités relevant de cette assistance humanitaire (logistique, eau, alimentation, santé, éducation...). Sur ce sujet, les meilleurs spécialistes de

\footnotetext{
${ }^{1}$ Ce conflit, très ancien, est à dissocier de l'actuelle crise du Darfour qui sévit dans l'Ouest du Soudan; mais, dans la situation politique complexe de ce pays, il serait très audacieux d'affirmer que cette autre guerre ne compromet pas l'issue des négociations de paix entre le Nord et le Sud.
}

la question présentent une vision particulièrement éclairante des contraintes et des limites de l'aide humanitaire, dès lors que cette dernière a souvent couvert - au nom de la souveraineté des États - l'impuissance des grandes puissances à mettre un terme à ces conflits (Brauman, 1996 ; Moore, 1998).

À la suite de la Conférence de Rio sur l'environnement en 1992, le HCR - comme toutes les agences des Nations unies - se mit en devoir d'intégrer la dimension environnementale en marge de ses activités statutaires d'assistance aux réfugiés. En Afrique, tout particulièrement, l'explosion du nombre de nouveaux conflits, ajoutés à ceux qui dataient de la guerre froide, provoqua des mouvements de population si massifs que la question de la dégradation du milieu consécutive à ces déplacements, et en premier lieu de la couverture végétale, devint un problème que le HCR pouvait d'autant moins évacuer que les États riverains des pays en guerre en tirèrent argument pour fixer leurs conditions à l'accueil des populations réfugiées.

Dans ce contexte, le HCR, dont le premier mandat reste la protection des réfugiés, mobilisa des compétences extérieures pour aborder cette question. Il le fit de deux façons : d'une part, en engageant un certain nombre de programmes de protection et de réhabilitation du milieu, d'éducation environnementale et d'économie d'énergie ; d'autre part, en lançant des études et des expertises visant à mesurer l'impact des populations réfugiées sur l'environnement et à en comprendre les mécanismes. Dans le cadre d'un projet concerté proposé par deux équipes du Cirad et de l'IRD $^{2}$, une approche commune fut retenue pour engager le HCR dans une démarche de « diagnostic et d'évaluation de la situation environnementale dans les régions d'accueil des populations réfugiées ». Face aux importantes controverses que suscitaient les superficies supposées affectées par les conséquences de ces afflux de population, le principe d'une approche à la fois intégrée et spatialisée de l'environnement finit par s'imposer au HCR.

Sans entrer dans les détails pratiques de cette coopération $^{3}$, on devine qu'il s'agissait moins, dans ce cas précis, d'un programme de recherche au sens strict que d'une opération d'assistance technique auprès du HCR et

\footnotetext{
${ }^{2}$ Cirad : Centre de coopération internationale en recherche agronomique pour le développement; IRD : Institut de recherche pour le développement.

3 De 1996 à 1998, une équipe de l'IRD participa à une première expérience de ce type dans les camps de réfugiés du Kenya. À la suite de cette collaboration, un financement du Fonds français pour l'environnement mondial (FFEM) permit à deux équipes du Cirad et de l'IRD d'engager des études similaires dans les deux pays retenus par le HCR : la Guinée (Cirad) et l'Ouganda (IRD). En Ouganda, ces travaux ont été conduits par A. Beaudou, L. Cambrézy et R. Zaiss.
} 


\section{Ouganda}

Localisation des sites agricoles

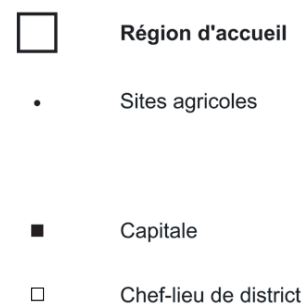

Frontière internationale

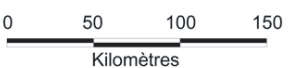

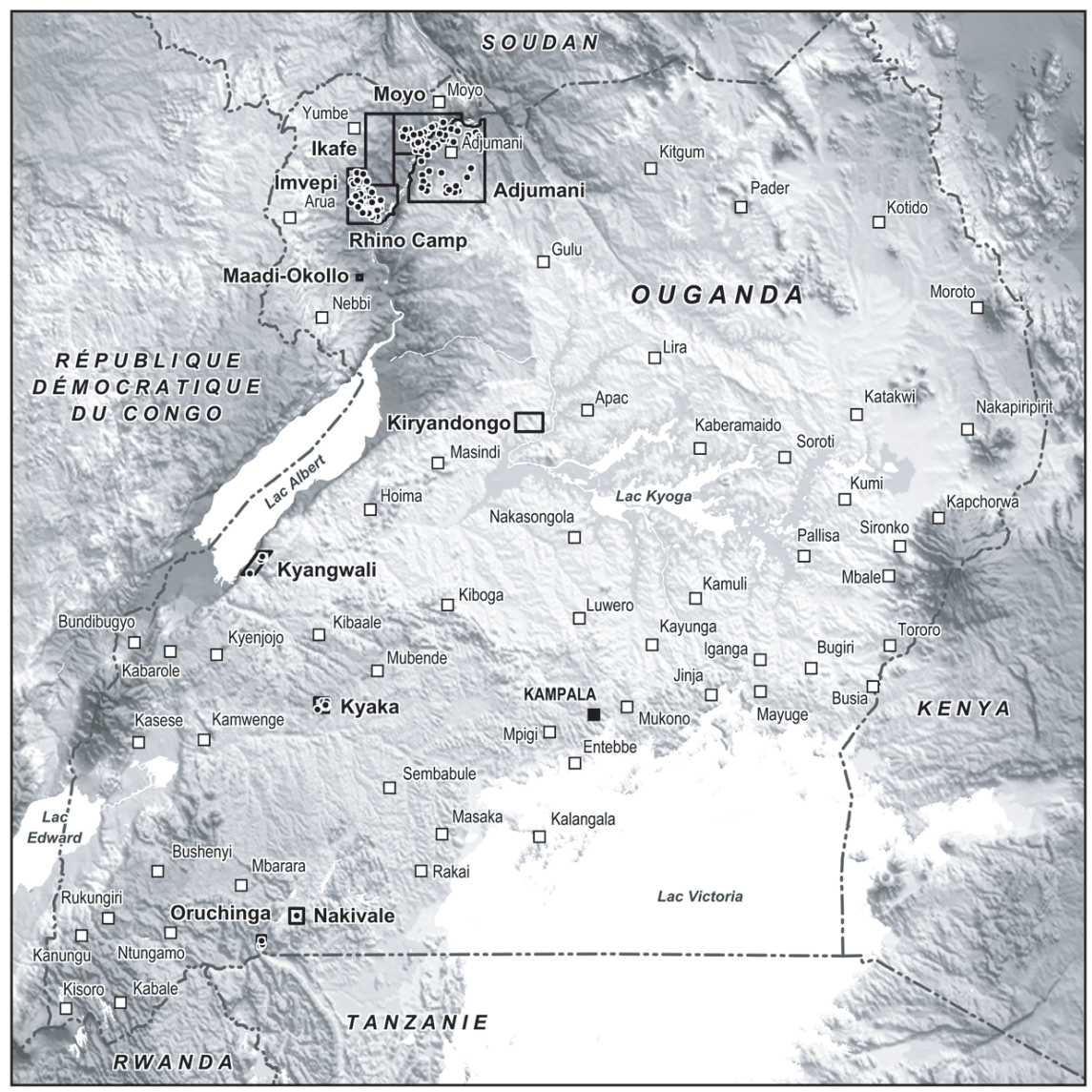

Source: base de données IRD/UNHCR

Fig. 1. Ouganda : principales zones d'accueil des réfugiés.

des ONG concernées ${ }^{4}$. Cependant, au-delà de la mise en œuvre des outils et des méthodes permettant de mesurer et de cartographier l'impact des réfugiés dans une région donnée, ce programme devait apporter une contribution à une question plus fondamentale qui, dans le cas des populations réfugiées, prend un relief tout particulier : comment gérer et aménager le territoire d'accueil de ces populations, et comment faire en sorte que la gestion de l'espace devienne une dimension intrinsèque de l'assistance humanitaire?

Les deux zones retenues dans le cadre de cette étude se situent au nord-ouest de l'Ouganda. Les deux secteurs choisis de Rhino Camp (district d'Arua) et d'Adjumani concentrent en effet le plus grand nombre de réfugiés et, corrélativement, le plus grand nombre de colonies agricoles (Fig. 1).

\footnotetext{
${ }^{4}$ L'approche SIG est coûteuse en temps : collecte des données, relevés au GPS (routes, parcellaire, limites administratives...), validation des images satellite, actualisation de la toponymie, etc., sont autant d'exercices obligés. Ce programme fut mené avec les ONG concernées. Cette proximité permit un accès aisé au terrain et aux enquêtes. Réciproquement, une formation des personnels à ces techniques fut dispensée, ainsi qu'un approvisionnement régulier en cartes thématiques et en images aériennes.
}

\section{Les guerres civiles dans le Nord de l'Ouganda et au Sud-Soudan : deux destins mêlés}

Comme pour son immense voisin du nord, le Soudan, la position géographique de l'Ouganda n'est pas sans importance dans les vicissitudes de son histoire. La position de ce pays, charnière entre différents ensembles géographiques et culturels, conduit à distinguer le Nord et le Sud de l'Ouganda. La moitié sud se rattache nettement à la région des Grands Lacs. Très densément peuplé, sur des sols riches et avec des précipitations abondantes, le Sud constitue le véritable poumon économique du pays. C'est aussi la région des « royaumes »-celui du Buganda est le plus connu - avec lesquels le colonisateur britannique avait négocié plus ou moins habilement la gestion administrative et politique du territoire (Calas et Prunier, 1994).

La moitié nord du pays, géographiquement et culturellement plus proche des zones frontalières du SudSoudan, connaît des conditions agroécologiques moins favorables; au nord-est, la faiblesse des précipitations explique d'ailleurs le caractère dominant de l'économie pastorale des Karamonjongs. Cette région - à l'exception 
notable des riches terres proches de la ville d'Arua, à la frontière avec la République démocratique du Congo (RDC) - est aussi, dans l'ensemble, beaucoup moins peuplée et les divers groupes ethniques qui la composent sont réputés pour être des sociétés "acéphales » ou peu structurées (Lacoste, 1995). À ces différences, s'ajoute l'obstacle naturel que constituent l'immense lac Kyoga et ses marais affluents. Orienté est-ouest, il contribue à cette fracture entre les deux entités : l'une, au sud, bien reliée à la capitale et en prise sur le développement ; l'autre, au nord, vivant l'isolement et le retard économique comme un abandon. De fait, un bon tiers du territoire national situé au nord du lac Kyoga se trouve ainsi très marginalisé et n'a guère profité des gains de croissance élevés des années 1990 (Torrenté, 1999). Malgré les désirs de paix d'un pays rendu exsangue après les régimes sanglants de Milton Obote et Idi Amin Dada, l'arrivée au pouvoir de Yoweri Museveni (un «sudiste») en 1986 provoqua la naissance d'un nouveau front interne aujourd'hui dirigé par Joseph Koni : le Lord Resistancy Army (LRA). D'origine acholi, principal groupe ethnique au nord du pays, ce mouvement aux contours idéologiques flous, mène une guérilla sans espoir mais d'une extrême violence contre le gouvernement Museveni. Sans véritablement menacer le pouvoir en place, les conditions d'insécurité sont telles dans les districts du Nord qu'elles ont provoqué la fuite de près de 1,6 millions de déplacés vers les centres urbains locaux (Gulu, Kitgum...). L'insécurité chronique d'une bonne partie du Nord du pays, associée au ressentiment et à la méfiance que ses populations inspirent à celles du Sud, ont largement contribué au creusement des écarts entre ces deux entités.

De l'autre côté de la frontière, le Soudan a connu entre 1983 et 2005 l'une des plus meurtrières et des plus longues guerres civiles qu'ait eu à supporter le continent africain. Là encore, la ligne de fracture passe entre le Nord et le Sud du pays. La domination musulmane du Nord, le refus au Sud d'un gouvernement régi par la charia, de puissants antagonismes religieux et ethniques entre les populations noires du Sud et arabes au Nord, ainsi qu'un accès contesté aux ressources pétrolières et au partage des revenus que son exploitation suppose sont quelques-uns des ingrédients de cette guerre civile. Le Sudan People Liberation Army (SPLA), bras armé de la rébellion sudiste, dirigé par le colonel Garang, bénéficia longtemps du soutien des pays riverains : l'Éthiopie, le Kenya (de façon plus discrète) et surtout l'Ouganda.

Ce soutien non dissimulé du gouvernement Museveni à la rébellion sudiste explique le paradoxe de l'appui offert par le gouvernement islamique de Khartoum à la guérilla d'inspiration chrétienne conduite par le LRA de Joseph Koni. Ainsi, chacun de ces deux mouvements rebelles trouvait dans le pays voisin les ressources matérielles et les bases arrière de repli assurant l'étonnante longévité de ces conflits et expliquant la difficulté des gouvernements en place à écraser cette opposition interne.

\section{Le choix des colonies agricoles pour l'accueil des réfugiés : limites et contraintes}

Les Soudanais réfugiés en Ouganda ont bénéficié d'un traitement particulier. En effet, contrairement à la situation la plus commune dans les pays d'accueil, où les réfugiés sont en général rassemblés dans des camps, en Ouganda, ils ont pu faire l'expérience des «colonies agricoles ${ }^{5} »$. Cette option consiste à attribuer temporairement des terres aux réfugiés de manière à ce que ces derniers renouent avec une activité productive, avec pour objectif affiché de parvenir à une certaine autosuffisance alimentaire. Les quelques 250000 réfugiés qu'accueille l'Ouganda sont répartis dans 68 colonies (soit une moyenne de 3700 réfugiés par site). Pour l'essentiel, ces colonies sont situées à l'extrême nord-ouest du pays, dans les districts d'Adjumani, Moyo et Arua, de part et d'autre du Nil (cf. Fig. 1).

L'option des colonies agricoles, retenue par le gouvernement, peut d'autant plus surprendre qu'un afflux massif et soudain de population étrangère suscite ou renforce en général une conception plutôt restrictive de l'accueil des immigrants et que celle-ci s'accompagne d'une forte volonté de contrôle des frontières et des mouvements de population. L'arrivée de réfugiés originaires d'un pays voisin est, en effet, non seulement perçue comme un événement qui, certes, oblige à respecter les engagements pris au regard de la convention de Genève, mais aussi comme une menace qui fait courir un certain nombre de dangers (réels ou supposés) pour le pays d'accueil. Le souci d'exercer un contrôle étroit sur ces flux de populations conduit habituellement les autorités qui en ont la charge à envisager des solutions qui, dans la pratique, par-delà l'obligation d'assistance, visent à restreindre la mobilité et la liberté d'action des réfugiés. C'est pourquoi, même si, ici ou là, des mesures sont adoptées pour assouplir ou rendre plus supportable la logique d'enfermement ou d'endiguement qui l'accompagne, le «camp de réfugiés » est et restera encore longtemps la principale modalité d'accueil des exilés (Cambrézy, 2001).

Dès lors, toute autre forme d'accueil que celle des camps devient sujet d'interrogation. En Afrique notamment, où les différents conflits ont provoqué la multiplication des camps aux frontières des pays en guerre, les

\footnotetext{
${ }^{5}$ L'expression consacrée en anglais pour ce type d'aménagements est rural settlements. La traduction littérale - d'ailleurs parfois utilisée - serait "sites agricoles». Dans le présent contexte, l'expression «colonies agricoles » peut être avantageusement employée.
} 
alternatives sont suffisamment rares pour mériter d'être signalées et étudiées (Lassailly-Jacob, 2003). L'Ouganda fait partie de ces exceptions, et, même si le rapatriement des réfugiés devient une hypothèse crédible, il n'est pas inutile d'expliquer les modalités de mise en œuvre de ces colonies ainsi que le contexte géographique et politique qui préside à ce type d'accueil.

On l'a dit, la solution des colonies agricoles consistant à attribuer des terres n'est pas sans risques. Car, si l'accueil des réfugiés est un devoir pour les États signataires de la convention de Genève, leur offrir des conditions très hospitalières est souvent (et à juste titre) considéré comme un acte inamical dirigé vers le régime en place dans le pays dont ils sont originaires. Par ailleurs, à côté de ces arguments de nature diplomatique, un certain nombre de raisons objectives rendent souvent cette solution difficile ou impossible. Ainsi, dans les régions où la pression démographique et foncière est importante, cette solution s'avère inapplicable faute d'espace disponible. Dans d'autres circonstances, ce sont les conditions naturelles qui interdisent cette option. Dans les régions désertiques ou très arides, par exemple, la faiblesse des précipitations interdit les cultures pluviales et le pastoralisme s'avère alors être l'activité dominante. Dans ces zones, l'équilibre entre les ressources naturelles et les charges de bétail est fragile. L'accès aux pâturages et aux points d'eau, sous contrôle de la population locale, rend inacceptable la perspective de voir les réfugiés franchir les frontières avec leur bétail. Autrement dit, pour accueillir des réfugiés dans des colonies agricoles, il faut non seulement le pouvoir, mais également le vouloir ; et cela signifie que les autorités du pays hôte - en fonction de cette alternative politique fondamentale - choisissent (ou non) une région d'accueil où peuvent se combiner volonté et possibilité d'établir les réfugiés dans de telles structures. On le voit, l'accueil des réfugiés dans un espace donné peut certes s'envisager comme une question de «capacité de charge » par rapport aux ressources naturelles et aux populations autochtones, mais c'est aussi une affaire de géopolitique; les modalités d'accueil des réfugiés (le camp versus la colonie agricole) en sont d'ailleurs une illustration.

Si les risques politiques sont importants, les bénéfices politiques de cette solution ne sont pas négligeables (Banégas, 1999). En affichant un respect plus affirmé des droits fondamentaux des réfugiés, ce choix s'avère d'autant plus une excellente opération diplomatique sur la scène internationale que, au-delà de la bonne image affichée à l'extérieur, l'option des colonies agricoles est censée réduire la dépendance des populations à l'égard de l'assistance humanitaire (et alimentaire en particulier). La communauté internationale qui finance l'essentiel de cette assistance - et toujours menacée par la «refugee fatigue » - ne peut voir que d'un très bon œil cette perspective.

\section{Entre vides et trop-pleins : l'inégale répartition géographique de la population en Ouganda et la logique d'implantation des colonies agricoles}

Dans le cas de l'Ouganda, les conditions nécessaires pour l'accueil des réfugiés dans des colonies agricoles se trouvaient réunies dans le Nord-Ouest du pays. Là, à distance raisonnable de la frontière avec le Soudan, d'importantes disponibilités en terre associées à des conditions agroclimatiques relativement favorables ont permis cette option. Ainsi, de part et d'autre du Nil, dans les savanes arborées et les forêts claires des districts d'Arua, de Moyo et d'Adjumani, les quelque 150000 réfugiés ont été répartis sur ce vaste espace dans plusieurs dizaines de colonies.

Pour comprendre la logique des choix qui président à ces implantations, l'inégale répartition de la population en Ouganda doit être rappelée. L'analyse des quatre cartes relatives aux densités de population entre 1960 et 1990 (Fig. 2) confirme un phénomène, finalement assez classique en Afrique, qui explique d'ailleurs largement le fait que ce continent demeure, jusqu'à nos jours, le moins urbanisé de la planète. Cet ensemble de cartes révèle en effet clairement un processus de diffusion progressive en tache d'huile du peuplement. Il se traduit par un double phénomène de densification des espaces préalablement investis et, de façon concomitante, de dispersion de la population par la colonisation des terres sur les marges encore inhabitées.

C'est en fonction de cette dynamique générale que l'installation des réfugiés dans les zones choisies par le gouvernement ougandais prend tout son sens. Ce phénomène de « remplissage » progressif des régions vides est ici à l'œuvre. La proximité d'aires plus peuplées, voire très denses (région d'Arua), fait des deux zones d'accueil des réfugiés (Rhino Camp et district d'Adjumani) des « déversoirs naturels » permettant d'absorber les excédents de population et ainsi de réguler la pression démographique et foncière de ces régions plus fortement peuplées.

Dans les deux grands ensembles étudiés (Rhino Camp et district d'Adjumani), les colonies agricoles ont été implantées sur des espaces encore vierges de toute occupation, à la périphérie de foyers de peuplement autochtone eux-mêmes encore relativement peu denses (de 25 à 50 habitants $/ \mathrm{km}^{2}$ ). Pour les sites les plus bas en altitude à proximité immédiate de la vallée du Nil, le déficit pluviométrique s'ajoute aux autres contraintes expliquant ces disponibilités en terre (Department of Lands and Surveys, Uganda, 1962). La topographie joue en effet un rôle sensible dans l'importance des précipitations, aussi bien dans le district d'Adjumani que dans celui d'Arua où se situent les colonies agricoles de Rhino Camp. Dans ce dernier cas, comme d'ailleurs dans toutes les zones proches du fleuve, la présence de la mouche tsé-tsé 

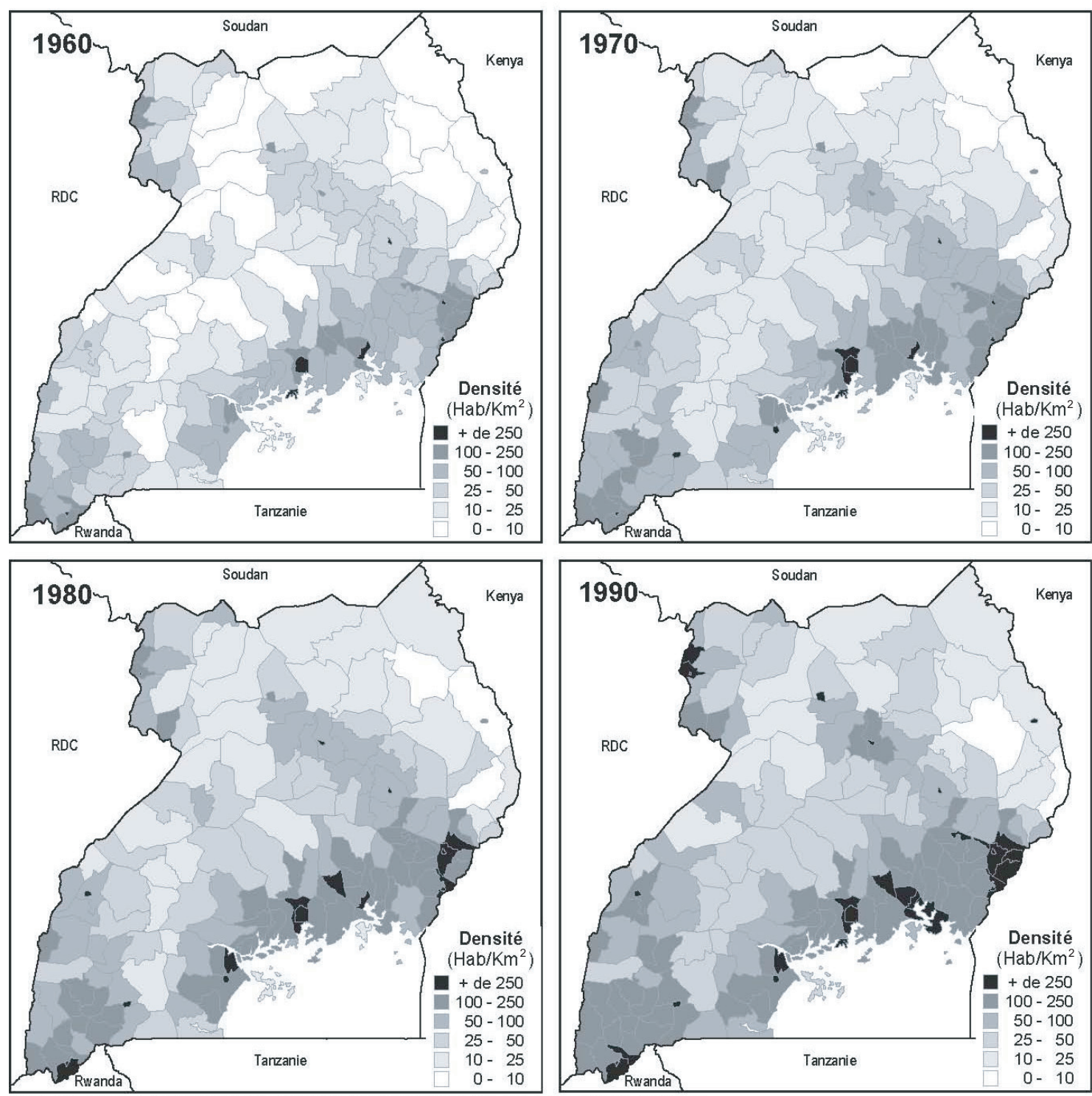

Source: UNEP (2001): Africa Population Database. Digital Chart of the World, First Edition 1993. IRD/UNHCR: FRAME Database.

Fig. 2. Ouganda : évolution des densités de population.

(éradiquée dans les années 1970) allait de pair avec celle d'une faune sauvage abondante. Cette configuration géographique particulière conduisit les colons britanniques à faire de cette région une réserve de chasse (d'où le nom de « Rhino Camp »).

Ainsi, l'ouverture de colonies agricoles pour les réfugiés s'apparente-t-elle largement à un front de colonisation (Fig. 3 et 4), même si, ici, l'objectif n'est pas de promouvoir une culture de rente. Dans le district d'Adjumani, en particulier, les colonies sont localisées en périphérie de la zone peuplée ${ }^{6}$. Le Sud du district

\footnotetext{
6 À Rhino Camp, 41 colonies agricoles ont été ouvertes entre 1994 et 1998 pour près de 25000 réfugiés, soit une colonie pour environ 600 réfugiés. La surface agricole attribuée en 2003 était de 3910 hectares. Dans le district d'Adjumani, 33 colonies ont été ouvertes entre 1987 et 2002. La superficie totale attribuée
}

reste encore vierge de toute occupation humaine et, à cet égard, l'ouverture de nouveaux espaces pour l'agriculture a évidemment pour corollaire le défrichement et la réduction de la couverture végétale, et tout particulièrement de la couverture arborée. Mais il est à signaler que le défrichement de la forêt claire ou de la savane boisée n'est nullement perçu comme un problème environnemental (Cambrézy, 2002). Nous ne sommes pas, ici, dans une logique - fréquente lorsqu'il s'agit de ce genre de population - où les réfugiés sont perçus et présentés comme un risque au regard de la protection de la végétation. Dans le contexte de systèmes agraires rudimentaires où seuls quelques arbres « utiles » sont préservés, prévaut la

(mesurée en 2003) était de 15854 hectares pour une population totale de 55379 réfugiés. 


\section{RHINO CAMP}

Distribution de la population par village en 2002
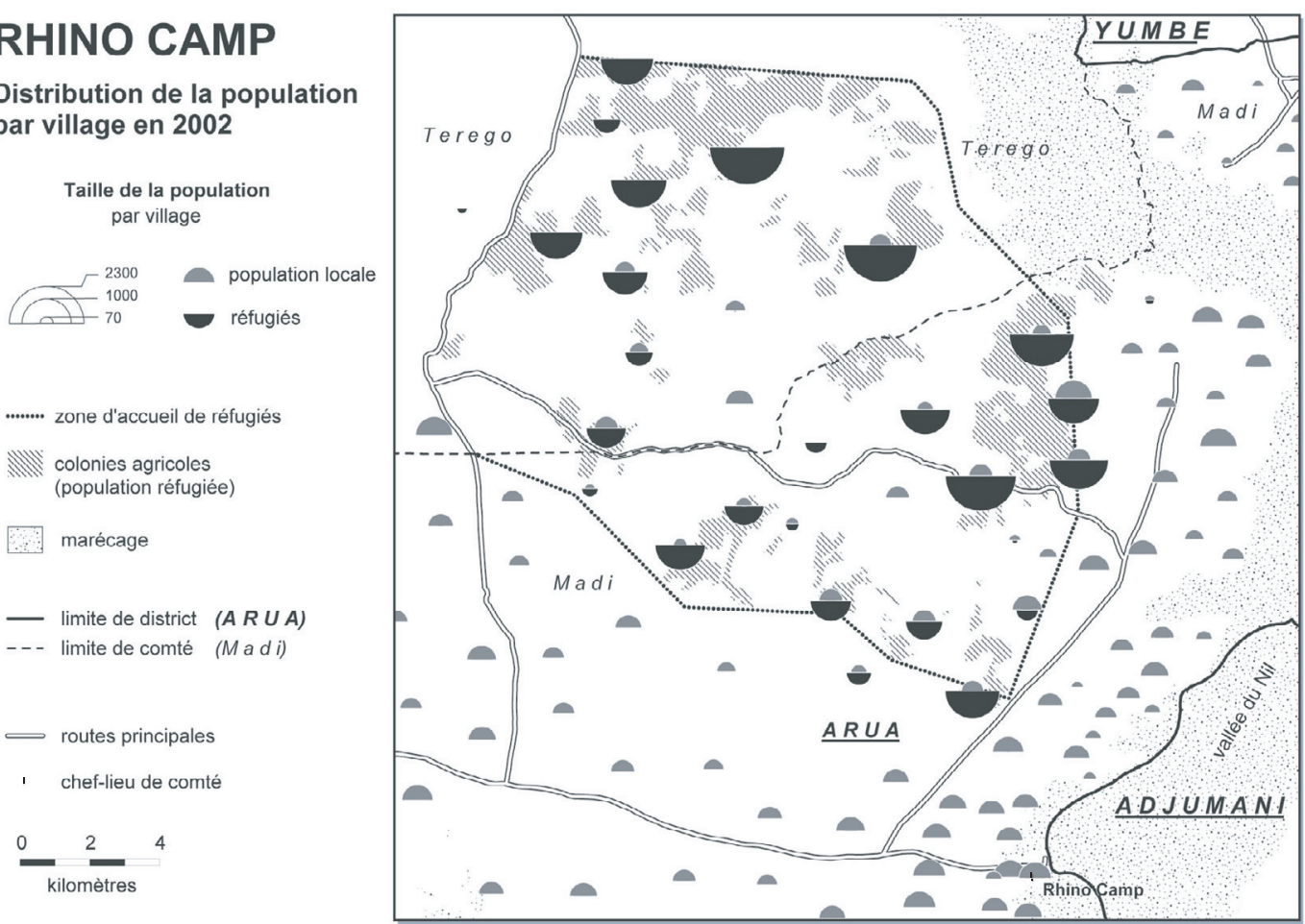

Sources : base de données IRD/UNHCR 2003. Réalisation : Rainer Zaiss IRD - LCA, Bondy, 2006

Fig. 3. Rhino Camp : distribution de la population par village en 2002.

\section{District Adjumani}

Distribution de la population par village en 2002

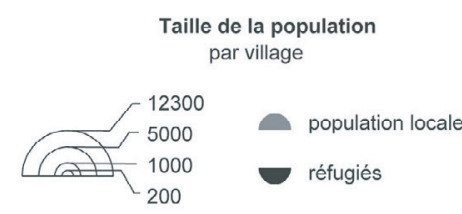

\section{colonies agricoles (population réfugiée) \\ Sud du district (espace non colonisé) \\ chef-lieu du district \\ routes principales}
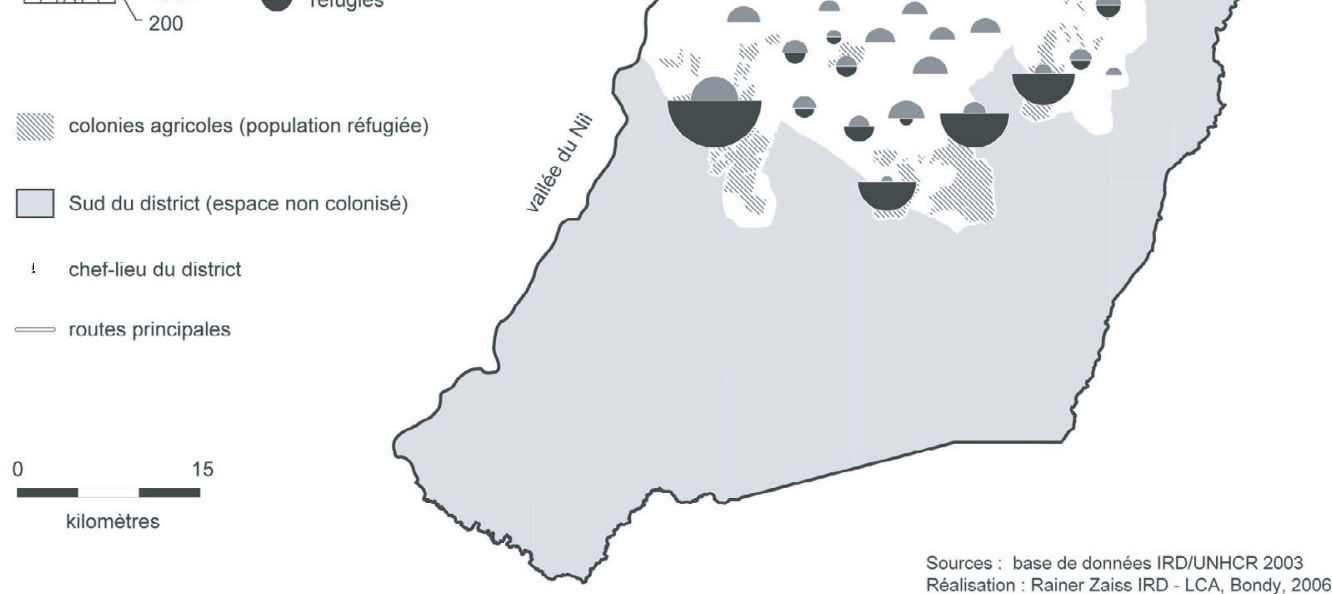

Fig. 4. District d'Adjumani : distribution de la population par village en 2002. 
logique la plus simple : pour ouvrir de nouveaux espaces à l'agriculture, il faut défricher et personne n'y trouve à redire.

\section{Les modalités pratiques d'organisation des colonies agricoles}

À l'instar de l'aide humanitaire dispensée dans les camps, en Ouganda, le HCR délègue de manière contractuelle aux organisations non gouvernementales (ONG) l'ensemble des programmes d'assistance : logistique, alimentation, santé, éducation, etc. (Haut Commissariat des Nations unies aux réfugiés, 2000). À ces tâches «habituelles ", s'ajoute, dans le cas des colonies agricoles, une activité moins usuelle s'agissant de réfugiés : l'attribution de terres et le soutien à la production agricole (outillage, semences, formation, etc.).

À raison d'une dotation théorique de 0,3 hectare par réfugié, deux types d'organisation de l'espace ont été retenus : 1) soit un peuplement dispersé où chaque famille est placée au cœur même du lopin de terre qui lui est attribué ; 2) soit un regroupement de la population dans un lieu unique (cluster) avec les parcelles de terre attribuées localisées à plus ou moins grande distance de ce village. Chacun de ces deux types présente des avantages et des inconvénients. L'avantage du premier est la proximité des terres de culture (et donc la réduction des temps de parcours pour l'accès aux champs). En revanche, dans un contexte de relative insécurité, le niveau de protection des réfugiés est faible et l'accès aux infrastructures communes (pompe, école, centre de santé) est très inégal. Dans le cas du regroupement des réfugiés dans un lieu unique, avantages et inconvénients sont exactement inverses.

Pour ce qui concerne l'attribution de terres, les ONG en charge des réfugiés ont dû procéder en deux étapes. Dans un premier temps, il s'agissait d'identifier - sous le contrôle et la supervision de "l'office du Premier ministre » et avec la participation active de la population locale ou de ses représentants - l'ensemble du territoire sur lequel les réfugiés pourraient recevoir un droit de culture. Dans un second temps, il fallait attribuer à chaque famille de réfugiés un lopin de terre d'une superficie établie en fonction du nombre de personnes recensées dans l'unité familiale $(0,2$ ou 0,3 hectare multiplié par le nombre de ses membres) $)^{7}$.

Il est à noter qu'à ces deux niveaux, les moyens techniques habituellement employés par le personnel des

\footnotetext{
7 Pour plus d'informations sur les résultats des études conduites par l'équipe IRD dans les sites de Rhino Camp et $\mathrm{du}$ district $\mathrm{d}$ 'Adjumani, voir http://www.bondy.ird.fr/carto/refugies/index.htm/ ainsi que http://www.bondy.ird.fr/carto/refugies/biblio.html
}

ONG ne permettaient pas de mesure précise des superficies. La portion de territoire attribuée pour l'installation d'une colonie agricole ne faisait l'objet d'aucune démarcation ni d'aucun bornage permettant d'en délimiter le périmètre. Il s'est par ailleurs avéré fréquent que les chefs de terre (landlords) accordent à des colons ougandais le droit de s'installer dans le périmètre "dévolu » aux réfugiés. Enfin, il n'était pas rare que les territoires alloués aux réfugiés soient situés dans des zones largement ou en partie incultes (terrains marécageux, escarpements ou affleurements rocheux, etc.). Dans ce cas, qu'il s'agisse d'une conséquence ou d'une stratégie volontaire de la part des populations locales, le manque de terres cultivables conduisait certains réfugiés à louer leur force de travail dans les exploitations des agriculteurs locaux.

$\mathrm{Au}$ total, le caractère très approximatif des modalités d'attribution des terres montre assez que, même si les réfugiés furent les bienvenus, il n'était pas pour autant question de laisser se créer les conditions permettant qu'ils acquièrent de quelconques «droits fonciers». Le refus de tout bornage, la crainte qu'inspiraient tous travaux de cartographie et l'installation de migrants «dans » le périmètre accordé aux réfugiés sont autant de faits qui témoignent de ce refus d'une installation durable des réfugiés. En outre, les levés de cartographie - qui avaient précisément pour objectif de délimiter les surfaces réellement attribuées - mirent en évidence le fait que le ratio de 0,3 hectare par réfugié était rarement atteint et que ces derniers devaient le plus souvent se contenter de 0,1 hectare.

Le double contrôle opéré à la fois par les chefs de terre et les représentants de l'État fixait ainsi clairement les limites politiques et foncières de cet accueil. Le défrichement et la mise en valeur de terres vierges par les réfugiés pouvaient certes donner à ces derniers un droit d'usage sur le sol, mais celui-ci n'était en aucune manière assimilable à un quelconque droit de propriété : l'installation restait provisoire et ne pouvait évidemment pas servir de prétexte à une revendication ultérieure de la nationalité ougandaise par le biais d'une sorte de «droit du sol».

Cette « générosité prudente » quant à l'allocation de terres aux réfugiés doit être lue dans le contexte de la réforme (en 1998) de la constitution ougandaise. En reconnaissant un droit de propriété sur les terres aux populations qui les exploitent ou qui y vivent, cette réforme engage l'Ouganda vers une privatisation progressive de la propriété foncière. Bien entendu, c'est d'abord en ville et dans les périphéries urbaines que le processus est le plus rapide. Dans les campagnes, notamment les plus reculées, de nombreuses années s'écouleront sans doute avant que les agriculteurs ne se trouvent dotés d'un titre de propriété en bonne et due forme.

Dans les régions étudiées, il semble pourtant quel'installation - même provisoire - des réfugiés contribue à 
accélérer le processus ou, tout au moins, à aiguiser les appétits de terres. C'est dans ce contexte que peut être interprétée l'extrême réticence des populations locales à voir s'effectuer une délimitation formelle du territoire dévolu à chaque site agricole comme des parcelles individuelles allouées aux réfugiés. Les populations locales le savent ou l'ont bien compris : borner, délimiter, cartographier sont souvent les préalables à l'appropriation d'un territoire. C'est sans doute aussi pour cette raison que l'on peut comprendre l'installation délibérée d'agriculteurs ougandais à l'intérieur même des périmètres dévolus aux réfugiés. Ces installations, qui ne peuvent en aucun cas s'effectuer sans l'accord du chef de terre et des autorités locales, est un signe très clair adressé tant aux réfugiés qu'aux organisations humanitaires chargées de leur attribuer des terres. La population locale est partout chez elle, les exilés ne seront jamais que des «invités temporaires".

\section{De l'aide aux réfugiés à l'aide au développement : une stratégie politique d'aménagement du territoire}

Dans la pratique, l'objectif affiché d'autosuffisance alimentaire pouvait d'autant moins être atteint qu'une application à la lettre de la règle de 0,3 hectare par réfugié autoriserait une densité de population théorique de 333 habitants $/ \mathrm{km}^{2}$ ! Ce constat ressortait régulièrement, aussi bien des enquêtes de consommation alimentaire conduites sous le contrôle du Programme alimentaire mondial (PAM) que des enquêtes d'estimation des récoltes effectuées chaque année par les ONG et les services en charge de ce secteur. Le dernier rapport en date du HCR confirme d'ailleurs cette observation répétée année après année. En 2005-2006, sur les 228000 Soudanais réfugiés en Ouganda, il est estimé que seulement « 40000 sont autosuffisants et ne sont pas assistés » (UNHCR, 2006) $)^{8}$. L'incertitude et l'insuffisance des précipitations de la première saison de culture, mais surtout l'exiguïté des surfaces effectivement attribuées, rendaient de fait cet objectif peu probable. En outre, sauf à imaginer que les « réfugiés-agriculteurs » soient approvisionnés en engrais, le maintien de la fertilité des sols (pour une production agricole stable et durable) passe par la pratique régulière de la jachère, et donc par une gestion des terres qui tienne compte de cette pratique consommatrice d'espace. Or, à aucun moment, cette question du maintien ou

\footnotetext{
${ }^{8}$ Les besoins estimés par le PAM en 2006 pour les réfugiés et les déplacés sont de 225000 tonnes de produits alimentaires (céréales, huile, haricots...), correspondant à une valeur de 131 millions de dollars. Les réfugiés bénéficiaires de cette aide sont estimés à 75000 (source: Projected 2006 Needs. World Food Programme, http://www.wfp.org/operations/current_operations/ project_docs/101211.pdf).
}

de la reconstitution de la fertilité des sols n'a été sérieusement envisagée. Par ailleurs, deux autres facteurs, récemment avancés par le HCR, contribuent à expliquer l'importante distance entre les objectifs annoncés et la réalité. D'une part, l'augmentation du nombre des réfugiés par le simple jeu de la croissance naturelle suppose une augmentation des demandes en terre et l'ouverture de nouvelles parcelles. D'autre part, l'extrême incertitude de la situation politique des deux côtés de la frontière contribue à un important va-et-vient des réfugiés. "Conséquence de ce contexte géopolitique, l'action envers les réfugiés en Ouganda doit prendre en compte ce constant mouvement de va-et-vient, avec de nouveaux arrivants et de nouveaux retours se déplaçant vers l'Ouganda ou en dehors à un rythme pratiquement quotidien » (UNHCR, 2006). La très forte mobilité d'une partie supposée importante de la population réfugiée s'accorde évidemment mal avec la stabilité que requiert l'activité agricole.

Cette mobilité est davantage constatée que mesurée ; il serait d'ailleurs bien difficile de le faire. Quelques chiffres donnent cependant une idée de la fragilité de la situation générale. Dans la perspective de l'accord de paix signé entre le SPLA et le gouvernement de Karthoum, le HCR envisageait en 2005 de rapatrier au Soudan 35000 réfugiés. Dans la pratique, du fait des multiples obstacles rencontrés au Soudan, ce chiffre a été réduit à 6000 . En 2006, le retour de 10000 réfugiés est attendu. Mais, depuis 2004, il est noté dans ce même document que 18000 nouveaux réfugiés originaires du Soudan sont arrivés en Ouganda... Les combats avec le LRA, les conflits interethniques et les crises alimentaires dont souffre le Sud-Soudan sont les principales raisons qui expliquent ces arrivées de population (ibid.).

Dans la mesure où une grande latitude est laissée aux réfugiés - y compris pour se livrer à des activités non agricoles -, il apparaît en fait clairement que la réussite de ce programme ne peut pas se mesurer (du point de vue du gouvernement) à la satisfaction de l'autosuffisance alimentaire. En d'autres termes, que les réfugiés réussissent ou non à tirer quelque chose du sol, force est de constater que la préoccupation première n'est pas là. À l'évidence, la solution des colonies agricoles répond davantage à des choix et à des intérêts politiques d'un autre ordre.

De manière très pragmatique, là où les conditions de sécurité rendaient ce projet envisageable - en particulier dans les districts du Nord-Ouest du pays -, le gouvernement ougandais a opté en réalité pour une stratégie de développement global en mettant à profit les moyens et le savoir-faire de l'assistance humanitaire délivrée aux réfugiés. Dès lors, c'est toute la problématique des réfugiés qui se trouve renversée. Au lieu de percevoir l'arrivée de ces derniers comme un fardeau (Harrel-Bond, 1986), le gouvernement a choisi d'en faire le fer de lance du développement. D'où l'idée, coûteuse mais apparemment 
efficace, de multiplier et de disperser les colonies agricoles. Mais de quel développement est-il ici question? En fait, essentiellement, de celui des infrastructures. Car cette stratégie répond à un objectif plus complexe qu'il n'y paraît.

En premier lieu, la multiplication des colonies et leur localisation en périphérie des régions déjà peuplées s'accompagne d'une ouverture de pistes qui marque le premier pas vers le désenclavement. Celui-ci est en effet essentiel pour passer d'une économie d'autosubsistance à une économie de marché. Pour pouvoir vendre des produits commercialisables (cultures de rente, charbon de bois, etc.), il faut pouvoir les écouler; d'où l'importance vitale pour le développement économique d'un réseau satisfaisant de voies de communication. Il n'était donc pas surprenant de voir les populations locales, encore isolées ou mal pourvues en termes de services, réclamer «leurs" réfugiés ${ }^{9}$. Car, ici, l'arrivée des réfugiés et de l'assistance humanitaire n'est pas seulement synonyme de routes et de pistes : c'est l'ensemble des besoins les plus essentiels (qui faisaient jusqu'alors défaut) qui devient soudainement accessible. Sachant que l'ouverture d'une colonie agricole entraîne la création non seulement de pistes d'accès, mais aussi d'écoles, de points d'eau et de centres de soin, on comprend en effet l'impatience des populations locales... Dans ce contexte, l'accès à la santé et à l'éducation, tout comme l'ouverture de pistes qui rattachent les zones les plus reculées aux routes principales et aux centres urbains, sont bien les conditions nécessaires - sinon les clés - du désenclavement, du développement et d'une insertion dans l'économie de marché.

De ce point de vue, la réussite de ce projet est incontestable. Les enfants vont à l'école, la population est soignée, l'eau potable, relativement accessible. Ici ou là naissent des petits centres de commerce et d'artisanat; les agriculteurs se lancent dans des cultures commerciales (le sésame en particulier) ou dans des productions faisant l'objet, en ville, d'une très forte demande (fruits, légumes, charbon de bois). Enfin, la forte présence du personnel humanitaire contribue à l'épanouissement d'un secteur tertiaire extrêmement dynamique (services, restauration, transports, banque.... $)^{10}$.

En second lieu, cette stratégie répond à une autre considération qui est loin d'être accessoire. La localisation des colonies agricoles s'inscrit également dans une

\footnotetext{
${ }^{9}$ Dans la perspective de nouvelles arrivées de réfugiés, il nous a été demandé à plusieurs reprises de procéder à la cartographie au GPS de nouvelles implantations. C'est au cours de ces opérations de délimitation des terres que les populations locales nous faisaient part de leur impatience de voir arriver ces réfugiés.

${ }^{10}$ L'aide humanitaire est d'ailleurs très visible. Le HCR ne manque aucune occasion de montrer - à l'aide de panneaux portant la mention «Donated by UNHCR »- l'ampleur de sa participation à la construction des diverses infrastructures.
}

politique globale de sécurisation du territoire. Dans le cas du district d'Adjumani, en particulier, l'ouverture des colonies agricoles réduit d'autant (en faisant «reculer la brousse ") les territoires vides dans lesquels le mouvement rebelle se dissimule. Cette situation, d'ailleurs parfois dénoncée par les réfugiés, place ces derniers en position de bouclier protecteur pour les populations locales. Mais c'est à leurs dépens, car, dans le cas des rares mais brutales attaques du LRA, les colonies agricoles sont en effet en première ligne.

\section{De l'accueil au droit d'habiter : la dimension géopolitique de l'aide aux réfugiés}

On l'a dit, l'expérience menée par l'Ouganda apporte un éclairage particulièrement intéressant dans la mesure où elle rappelle qu'un peuplement suffisant des régions marginalisées est bien entendu une condition du développement de ces territoires. En effet, pour d'évidentes nécessités d'économie d'échelle, le sous-peuplement ne fait pas bon ménage avec le développement des infrastructures. Car on n'ouvre pas aisément des routes, des écoles ou des centres de santé dans un espace sous-peuplé et trop isolé de régions plus dynamiques. Des densités de population trop faibles dans des régions trop éloignées et trop mal connectées aux centres de décision sont, classiquement, des phénomènes qui se conjuguent, font système et creusent inexorablement les écarts de développement entre ces marges et les régions les plus dynamiques.

Bien sûr, on peut douter de la solidité de cette forme de développement dès lors que le désenclavement géographique, économique et social de ces régions est associé à une assistance humanitaire liée à la présence conjoncturelle de réfugiés. La question est ouverte et mérite d'être approfondie; elle interroge autant l'économie du développement que l'aide humanitaire. Or, entre l'assistance humanitaire et l'aide au développement, c'est un peu la même question qui est posée : «[...] pour éviter de réduire pour l'avenir toute organisation sociale à la distribution de secours » (Chemillier-Gendreau, 1996), on ne peut rendre de meilleur service aux réfugiés qu'en cherchant à dépasser la seule logique de l'assistance.

Au-delà des enseignements, en matière de développement, que livre le cas de l'Ouganda sur cette autre façon $\mathrm{d}$ 'accueillir les réfugiés, le choix des lieux et la manière dont ils sont investis révèlent la dimension géopolitique et souvent peu lisible de l'assistance auprès des réfugiés - un point $\mathrm{d}^{\prime}$ ailleurs singulièrement absent de la convention de Genève.

Pour le bon déroulement de son action, l'assistance humanitaire doit d'abord tenir compte du postulat ethnonational de départ selon lequel le droit à une terre ou à un espace de vie est subordonné à la division politique des États, laquelle fonde le droit d'accès au territoire et 
détermine la nationalité de ses habitants. Dans le contexte du Nord-Ouest de l'Ouganda, on a vu que les conditions se sont trouvées réunies pour que chacune des parties trouve son compte à cette forme originale d'assistance humanitaire. Ce faisant - avec des intentions ou des attentes évidemment différentes -, la nécessaire négociation, entre les réfugiés, la population locale, le gouvernement ougandais et les organisations humanitaires, contribue à éclairer la question fondamentale des articulations difficiles et complexes entre l'autochtone et l'étranger, d'une part, entre la souveraineté nationale et la " communauté internationale », d'autre part. De ce point de vue, le cas de figure présenté ici s'inscrit comme un élément de réflexion à ajouter au dossier, plus large encore, des migrations internationales.

Une manière d'approfondir cette question consiste à se placer dans l'hypothèse (tout à fait d'actualité) du rapatriement des réfugiés dans leurs régions d'origine au Soudan. Bien que ce retour s'annonce long et difficile en raison des incertitudes qui continuent de peser sur le processus de paix engagé entre les «sudistes » et le gouvernement de Khartoum, l'hypothèse d'une fermeture complète des colonies agricoles, consécutive au départ des réfugiés, ne peut être envisagée sans quelques inquiétudes par les autorités ougandaises. En effet, dans la mesure où le développement de la région de Rhino Camp, et plus encore du district d'Adjumani, a été rendu possible par la manière dont a été conçu l'accueil des réfugiés, il est évidemment à craindre que le départ de ces derniers n'entraîne à nouveau ces espaces périphériques dans la spirale du sous-développement. Cette hypothèse est d'autant plus envisageable qu'il s'agit de deux espaces qui, sans les réfugiés, risqueraient de retomber dans la marginalité économique et sociale. Dans une région globalement très troublée, elle semble politiquement peu supportable et conduit à se poser deux questions : le gouvernement ougandais a-t-il les moyens matériels, humains et financiers de compenser le «manque à gagner » que constituerait la fin des programmes d'assistance humanitaire? Et, indépendamment de l'origine de ces financements qui resteront de toute façon nécessaires, le départ des réfugiés est-il préférable à une politique assumée d'intégration définitive de ces populations sur le sol ougandais?

Dans l'immédiat, il serait très aventureux de prédire ce que deviendront ces deux régions. Sans préjuger des intentions des diverses populations réfugiées (comme des autres acteurs), il est clair qu'il y a là un terrain et une problématique de recherche qui mériteraient des études ultérieures. Après tout, les colonies de peuplement fondées sur une immigration étrangère ne sont pas des situations si fréquentes. Et, de ce fait, la recherche gagnerait beaucoup à investir davantage dans la problématique insuffisamment travaillée du rapport entre migrations internationales et territoire. Car, au fond, c'est bien la question que pose toute présence de réfugiés. Au-delà $\mathrm{du}$ droit d'asile et de la protection auxquels la convention de Genève est censée répondre, de quel «droit du sol » les réfugiés disposent-ils ? Ou, pour le dire autrement, à quel «droit d'habiter » peuvent-ils prétendre?

\section{Références}

Balancie, J.-M., de la Grange, A., 1999. Mondes rebelles : l'encyclopédie des conflits, Paris, Michalon.

Banégas, R. (Ed.), 1999. Ouganda, une puissance régionale?, Politique africaine, 75.

Brauman, R., 1996. L'Action humanitaire, Paris, Flammarion.

Calas, B., Prunier, G., 1994. L'Ouganda contemporain, Paris, Karthala.

Cambrézy, L., 2001. Réfugiés et exilés : crise des sociétés, crise des territoires, Paris, Éditions des Archives contemporaines.

Cambrézy, L., 2002. Espace, environnement et développement. Du bon usage des populations réfugiées : 1'accueil des exilés soudanais en Ouganda, in Martin, J.-Y., Le Développement durable : doctrines, pratiques, évaluation, Paris, IRD Éditions.

Chemillier-Gendreau, M., 1996. Portée et limites de l'ingérence humanitaire en Afrique, Afrique contemporaine, 180, 229-241.

Department of Lands and Surveys, Uganda, 1962. Atlas of Uganda, Entebbe.

Harrel-Bond, B.E., 1986. Imposing Aid: Emergency Assistance to Refugees, Oxford, Oxford University Press.

Haut Commissariat des Nations unies pour les réfugiés, 2000. Les Réfugiés dans le monde : cinquante ans d'action humanitaire, Paris, Autrement.

Lacoste, Y. (Ed.), 1995 [1 $1^{\text {re }}$ éd. 1993]. Dictionnaire de géopolitique, Paris, Flammarion.

Lassailly-Jacob, V., 2003. Conditions d'accueil et insertion des réfugiés dans un pays d'Afrique australe : la Zambie, in Guillon, M., Legoux, L., Ma Mung, E. (Eds), L'Asile politique entre deux chaises, Paris, Karthala.

Lavergne, M. (Ed.), 1989. Le Soudan contemporain, Paris, Karthala.

Moore, J. (Ed.), 1998. Des choix difficiles : les dilemmes moraux de l'humanitaire, Paris, Gallimard.

Torrenté, N. de, 1999. L'Ouganda et les bailleurs de fonds, Politique africaine, 75, 72-90.

UNHCR, 2006. Country Operations Plan - Uganda. Rapport annuel HCR, Genève.

Reçu le 10 avril 2005. Accepté le 6 avril 2006. 